\title{
BIOESTRATIGRAFÍA BASADA EN QUISTES DE DINOFLAGELADOS DEL NEÓGENO EN EL NORESTE DE LA PATAGONIA, ARGENTINA
}

\author{
SABRINA N. FUENTES \\ Museo Argentino de Ciencias Naturales "Bernardino Rivadavia". Av. Ángel Gallardo 470, C1405DJR, \\ Ciudad Autónoma de Buenos Aires, Argentina.sfuentes@macn.gov.ar \\ M. VERÓNICA GULER \\ Instituto Geológico del Sur (INGEOSUR), CONICET, San Juan 670, B8000ICN, Bahía Blanca, \\ Buenos Aires, Argentina.vguler@criba.edu.ar
}

JOSÉ I. CUITIÑO

Instituto Patagónico de Geología y Paleontología (CCT CENPAT-CONICET). Boulevard Brown 2915, U9120ACD, Puerto Madryn, Chubut, Argentina.jcuitiño@cenpat-conicet.gob.ar

\section{LUIS PALAZZESI}

Museo Argentino de Ciencias Naturales "Bernardino Rivadavia", CONICET, Av. Ángel Gallardo 470, C1405DJR, Ciudad Autónoma de Buenos Aires, Argentina.lpalazzesi@macn.gov.ar

ROBERTO A. SCASSO

Departamento Ciencias Geológicas, FCEyN C1428EHA, Universidad de Buenos Aires, CONICET. rscasso@gl.fcen.uba.ar

VIVIANA D. BARREDA

Museo Argentino de Ciencias Naturales "Bernardino Rivadavia”, CONICET, Av. Ángel Gallardo 470, C1405DJR, Ciudad Autónoma de Buenos Aires, Argentina.vbarreda@macn.gov.ar

\begin{abstract}
BIOSTRATIGRAPHY BASED NEOGENE DINOFLAGELLATE CYSTS IN NORTHEAST OF PATAGONIA, ARGENTINA. This paper presents the biostratigraphic results based on dinoflagellate cysts from two Neogene lithostratigraphic units cropping out in northeastern Patagonia. The Barranca Final Formation, located in the southwestern Colorado Basin, is exposed along the northern coast of San Matías Gulf, Río Negro Province, and the Puerto Madryn Formation in the Península Valdés, Chubut Province. The low-to-moderate diverse dinoflagellate cyst assemblages present are dominated by neritic taxa with subordinate oceanic indicators. Towards the top of both sections, the relative frequencies of the dinoflagellate cyts decrease, whereas acritarchs and continental palynomorphs proportions increase, indicating a shallowing upwards trend. The stratigraphic range of Labyrinthodinium truncatum truncatum in the lower part of the Barranca Final Formation indicate age between the late Burdigalian ( $c a .16 .5 \mathrm{Ma}$ ) and the late Tortonian ( $c a .7 .5 \mathrm{Ma}$ ) time interval. Labyrinthodinium truncatum truncatum and Habibacysta tectata constrain the age of the Puerto Madryn Formation to the Serravalian to Tortonian interval (late middle miocene to early late Miocene). Theses ages are consistent with the ${ }^{87} \mathrm{Sr} /{ }^{86} \mathrm{Sr}$ isotopic analysis of the oyster valves at 9.61 and 8.3 and with the ${ }^{87} \mathrm{Sr} /{ }^{86} \mathrm{Sr}$ ratios on calcitic shells at about $10 \mathrm{Ma}$, respectively.
\end{abstract}

Key words: dinoflagellate cysts, Miocene, Northern Patagonia, Argentina.

RESUMO - O presente artigo inclui resultados bioestratigráficos baseados em cistos de dinoflagelados de duas unidades litoestratigráficas do Neógeno que afloram no nordeste da Patagônia. A Formação Barranca Final, localizada no sudoeste da Bacia do Rio Colorado, que está exposta ao longo da costa norte do Golfo San Matías, Província de Rio Negro, e a Formação Puerto Madryn na Península Valdés, Província de Chubut. Os conjuntos de dinocistos das seções estudadas exibem uma diversidade moderada a baixa, composta principalmente dos táxons neríticos, com indicadores oceânicos, como Nematosphaeropsis rigida e Impaginidium ssp., sendo apenas subordinados. As frequências relativas de cistos decrescem na porção superior de ambas as secções, enquanto os acritarcas e palinomorfos continentais aumentam suas proporções, indicando uma tendência à redução da profundidade. $\mathrm{O}$ intervalo estratigráfico de Labyrinthodinium truncatum truncatum na parte inferior da Formação Barranca Final indica idade entre o final do Burdigaliano (ca. 16,5 Ma) e o Tortoniano final (cerca de 7,5 Ma). Labyrinthodinium truncatum truncatum, Habibacysta tectata restringe a idade da Formação Puerto Madryn ao intervalo entre o Serravaliano ou Tortoniano (final do Mesomioceno ao Neomioceno). Essas idades são consistentes com as análises isotópicas ${ }^{87} \mathrm{Sr} /{ }^{86} \mathrm{Sr}$ das conchas de ostras que resultam em valores de 9,61 e 8,3 e com as proporções de ${ }^{87} \mathrm{Sr} /{ }^{86} \mathrm{Sr}$ em conchas calcíticas de uns $10 \mathrm{Ma}$, respectivamente.

Palavras-chave: cistos de dinoflagelados, Mioceno, nordeste da Patagonia, Argentina. 


\section{INTRODUCCIÓN}

Durante el Neógeno, extensas áreas de Argentina fueron cubiertas por un mar somero conocido en la literatura como mar "Entrerriense" o "Paranaense" (Frenguelli, 1921; Feruglio, 1949; del Río \& Martínez, 1998; del Río, 2000; Marengo, 2015), cuyos depósitos han sido definidos como diferentes unidades litoestratigráficas en varias cuencas sedimentarias. En el noreste de la Patagonia, depósitos marinos neógenos fueron definidos como Formación Puerto Madryn (Haller, 1979) en el ámbito de la Cuenca de Valdés, y como Formación Barranca Final en el ámbito de la Cuenca del Colorado (Figura 1) (Kaasschieter, 1963, 1965). La mayor parte de la columna sedimentaria de la Cuenca del Colorado se reconoce únicamente en subsuelo y la Formación Barranca Final constituye una de las pocas unidades sedimentarias aflorantes.

La edad de los depósitos de la transgresión Entrerriense en el noreste de la Patagonia ha sido definida como Mioceno tardío basada principalmente en su contenido fosilífero (Feruglio, 1949; del Río, 2004; Palazzesi \& Barreda, 2004; Cione et al., 2005; Dozo et al., 2010). Dataciones por el método ${ }^{87} \mathrm{Sr} /{ }^{86} \mathrm{Sr}$ han dado valores cercanos a los 10 Ma para la Formación Puerto Madryn (Scasso et al., 2001) y entre 9 y 6 Ma para la Formación Barranca Final (Palazzesi et al.,
2014), las que permiten calibrar la edad de los depósitos sobre los cuales se efectúan estos estudios palinológicos.

Ha sido reconocida la importancia bioestratigráfica de los quistes de dinoflagelados en las últimas décadas (e.g. Stover et al., 1996; Williams et al., 2004), constituyendo actualmente una herramienta de alta resolución en la exploración de hidrocarburos. Además, los quistes de dinoflagelados son sensibles a los cambios de los parámetros físico-químicos de las masas de agua superficiales, siendo muy útiles para las reconstrucciones paleoambientales, particularmente con respecto a la productividad, salinidad y temperatura del agua superficial, así como también indican distancia a la línea de costa, transporte y migración (e.g. Wall et al., 1977; Pross \& Brinkhuis, 2005; Sluijs et al., 2005).

El presente trabajo documenta la distribución de los quistes de dinoflagelados en dos sucesiones sedimentarias neógenas que afloran en el noreste de Patagonia y propone una detallada comparación entre ambas. Por un lado se estudian las asociaciones de los quistes de dinoflagelados de la Formación Barranca Final en su sección tipo, provenientes de un nuevo muestreo palinológico vinculado a dataciones ${ }^{87} \mathrm{Sr} r{ }^{86} \mathrm{Sr}$ (Palazzesi et al., 2014). Además, se presentan los primeros registros de los quistes de dinoflagelados de la Formación Puerto Madryn, también asociados a edades ${ }^{87} \mathrm{Sr} /{ }^{86} \mathrm{Sr}$ (Scasso et al., 2001).

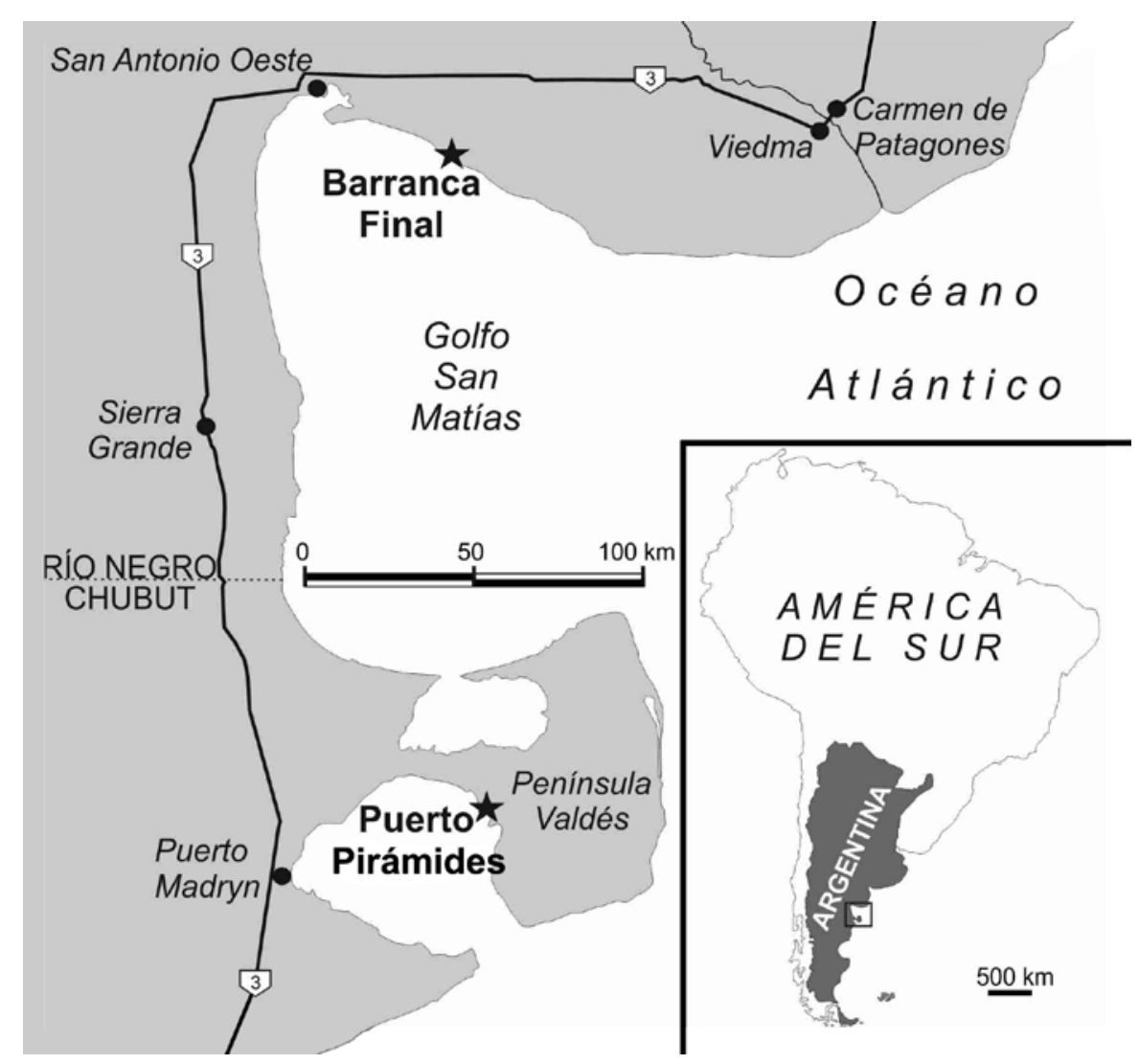

Figura 1. Mapa de ubicación mostrando la posición de la Formación Puerto Madryn en la sección Puerto Pirámides y la Formación Barranca Final en su sección tipo.

Figure 1. Location map showing the position of the Puerto Pirámides section of the Puerto Madryn Formation and the type section of the Barranca Final Formation. 


\section{GEOLOGÍA DEL ÁREA DE ESTUDIO}

Los depósitos neógenos marinos se encuentran ampliamente distribuidos en el noreste de la Patagonia, aunque sus espesores son relativamente delgados y las exposiciones se encuentran restringidas fundamentalmente a los acantilados costeros. En esta región se distinguen dos zonas de acumulación neógenas correspondientes a la Cuenca de Valdés en el noreste de Chubut y la Cuenca del Colorado, cuyos depocentros se encuentran costa afuera en el atlántico Sudoccidental (Zambrano, 1980). Hacia el oeste, los depósitos neógenos marinos se acuñan sobre el Macizo Norpatagónico, el cual fue un área positiva durante esta sedimentación. La Formación Puerto Madryn aflora fundamentalmente en la Península Valdés y en los alrededores de la ciudad de Puerto Madryn (Haller, 1979; Figura 1) y consiste de areniscas, heterolitas y capas bioclásticas, depositadas en un ambiente marino a estuarino con marcada influencia de mareas (Scasso \& del Río, 1987; del Río et al., 2001; Scasso et al., 2012) (Figura 2). Hacia el oeste la unidad se acuña (Feruglio, 1949; Scasso \& del Río, 1987), mientras que hacia el este se han reportado hasta 300 metros de facies similares en el subsuelo del oriente de la Península de Valdés (Masiuk et al., 1976; Caramés et al.,
2004). La Formación Barranca Final (Kaasschieter, 1965) ha sido descripta fundamentalmente en subsuelo con espesores de 265 a $772 \mathrm{~m}$, aunque es probable que este espesor incluya depósitos asignables a la transgresión "Patagoniense" del Mioceno temprano (Malumián et al., 1998). Sus únicos afloramientos, se encuentran en la costa norte del Golfo de San Matías (Figura 1), con aproximadamente $15 \mathrm{~m}$ de potencia. Allí la unidad se compone de pelitas laminadas y escasas areniscas finas intercaladas, con algunos niveles fosilíferos (Gelós et al., 1993; Malumián et al., 1998; Guler et al., 2002; Palazzesi et al., 2014), depositadas en ambiente marino a marino-marginal (Guler et al., 2002; Palazzesi et al., 2014). Su base no está expuesta y su tope es transicional hacia los depósitos continentales de la Formación Río Negro (Palazzesi et al., 2014).

\section{MATERIAL Y MÉTODOS}

Las muestras para el análisis palinológico provienen de los depósitos más finos, pelíticos, de las formaciones Puerto Madryn (42 $34^{\prime} 39^{\prime \prime}$ 'S $\left.64^{\circ} 16^{\prime} 35^{\prime \prime} \mathrm{O}\right)$ y Barranca Final (4054'38'S; 64²5'14"O). Se estudiaron 12 muestras de la sección inferior del afloramiento de la Formación Barranca
SECCIÓN BARRANCA FINAL

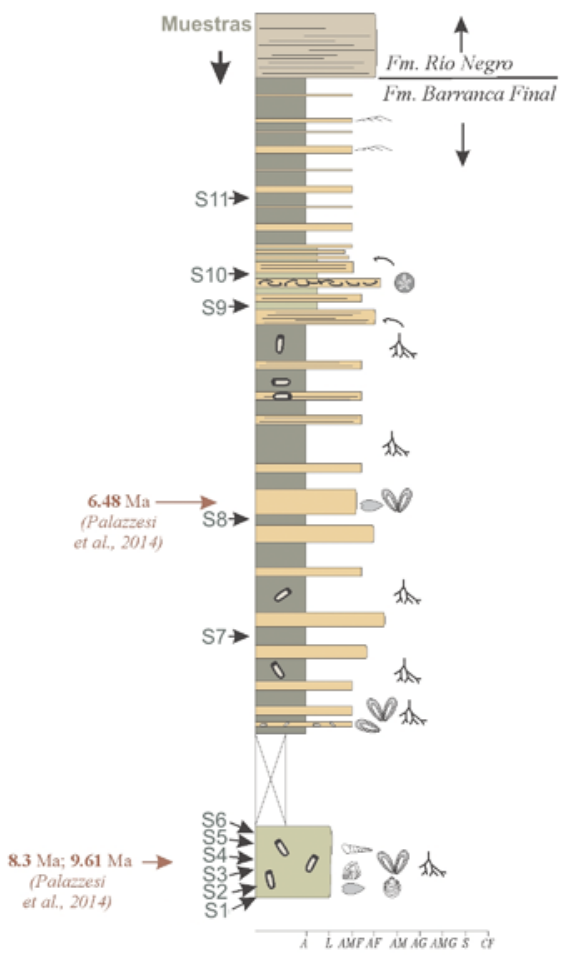

SECCIÓN PUERTO PIRÁMIDE

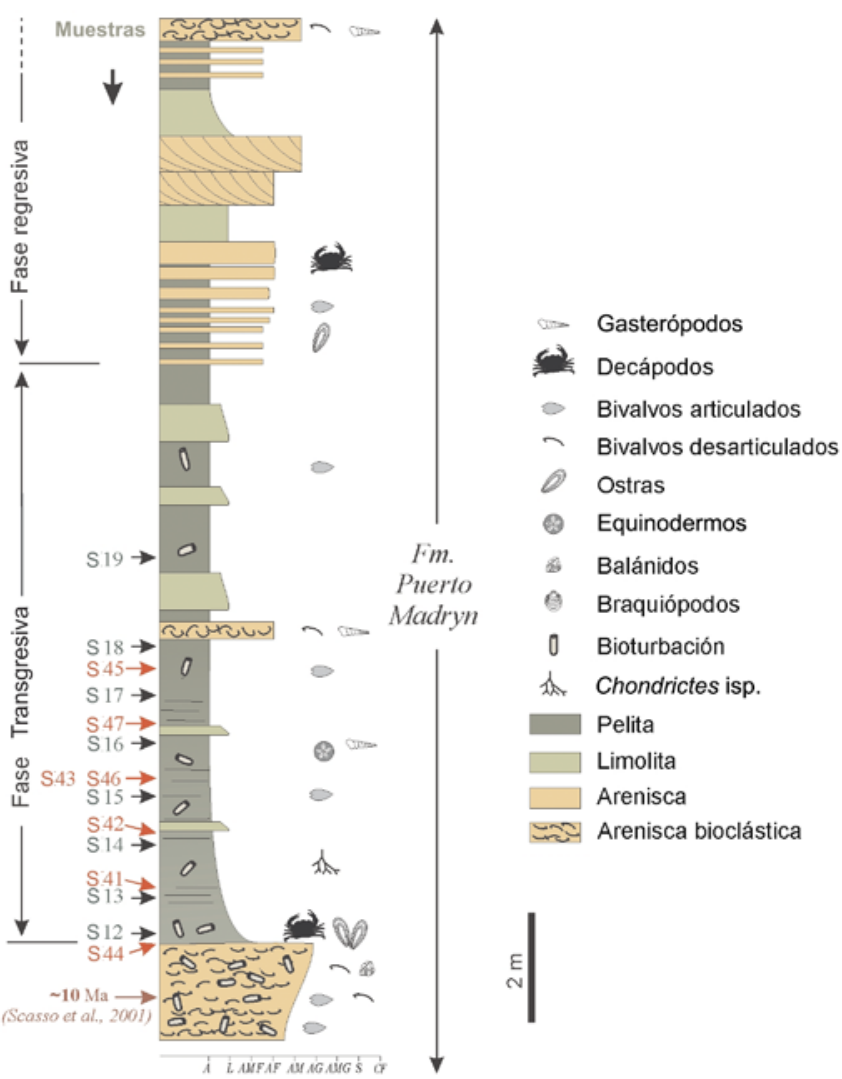

Figura 2. Litología, ubicación de las muestras, edades radiométricas y características paleontológicas de la Formación Puerto Madryn en la sección Puerto Pirámides y la Formación Barranca Final en su sección tipo (modificado de Palazzesi et al., 2014).

Figure 2. Lithology, position of the samples, radiometric ages and paleontological characteristics of the Puerto Pirámides section of the Puerto Madryn Formation and the type section of the Barranca Final Formation (modified from Palazzesi et al., 2014). 
Final en su localidad tipo (Figura 2) y 15 de la sección inferior del afloramiento de la Formación Puerto Madryn en las inmediaciones de la localidad de Puerto Pirámides (Figura 2), las cuales resultaron palinológicamente productivas. Las muestras S12-S19 de la Formación Puerto Madryn, se obtuvieron en un primer muestreo para el estudio de los palinomorfos continentales (e.g. Palazzesi et al., 2014) у las muestras S41-S47 (flechas) corresponden a un segundo muestreo de niveles similares.

El procesamiento físico-químico de las muestras consistió en la eliminación de los componentes inorgánicos, principalmente carbonatos y silicatos, con $\mathrm{HCl}$ y $\mathrm{HF}$, respectivamente. Los residuos fueron filtrados con mallas de 10 y $25 \mu \mathrm{m}$, teñidas con safranina y montadas en glicerina gelatina. Los quistes de dinoflagelados fueron analizados con un microscópio óptico de luz transmitida Nikon Eclipse 600. Fueron calculadas y representadas las frecuencias relativas de las especies de quistes de dinoflagelados, algas y acritarcos utilizando el programa Tilia 2.0.38 (Grimm, 1991-2014). Adicionalmente, se contaron hasta 250 especímenes por muestra, para calcular la relación entre palinomorfos marinos y continentales $(M / C=M /(M+C)$ y se representaron las frecuencias relativas de los quistes de dinoflagelados Gonyaulacales $(\mathbf{G})$ y Peridiniales $(\mathbf{P})$ sobre el total de quistes de dinoflagelados $(\mathrm{G} / \mathrm{P}=\mathrm{G} /(\mathrm{G}+\mathrm{P})$ ) (Figuras 3-4). Los especímenes ilustrados se tomaron con una cámara digital Nikon Coolpix 950. La taxonomía de los quistes de dinoflagelados corresponde a Fensome \& Williams (2004) y la escala geocronológica corresponde a Gradstein et al. (2012).

\section{COMPOSICION DE LAS ASOCIACIONES DE QUISTES DE DINOFLAGELADOS}

\section{Formación Barranca Final en su sección tipo}

Se identificaron y cuantificaron 19 especies de quistes de dinoflagelados, algas clorofíceas (especies de Botryococcus sp., Pediastrum sp.) y algas prasinoficeas (Tasmanites sp.) en la sección tipo de la Formación Barranca Final (Figuras $3,5-6)$. Los palinomorfos presentan en general un grado óptimo de preservación y las cuatro muestras inferiores (S1S4) contienen la máxima abundancia y riqueza de quistes de dinoflagelados (Figura 3). Estas muestras están dominadas por especímenes de Operculodinium centrocarpum, Brigantedinium simplex y $B$. cariacoense, agrupados en los recuentos como Brigantedinium spp., Lingulodinium hemicystum y diferentes especies de Spiniferites (S. mirabilis, S. ramous y Spiniferites/Achomosphaera sp.) (Figura 3). Las asociaciones están dominadas por especies del orden Gonyaulacales, mientras que las del orden Peridiniales están representadas únicamente por la familia Protoperidiniaceae, entre ellos Brigantedinium spp. y las especies S. brevispinosa brevispinosa, S. quanta, S. nephroides y $S$. dionaeacysta (Figura3). Las frecuencias relativas de las especies protoperidiniaceas son bajas, sin embargo están presentes en casi todas las muestras a lo largo de la sección. Las muestras ubicadas desde la parte media hacia el tope de la sección (S5 a
S12), representan una disminución en la abundancia y riqueza de quistes de dinoflagelados, quedando reducidas a un total de cuatro especies en las dos muestras superiores (Figura 3). Las algas clorofíceas representadas por Botryococcus sp. y Pediastrum sp. así como también las algas prasinofíceas representadas por Tasmanites sp. predominan en dicho intervalo. Estas últimas muestran un incremento notable en la muestra S7 constituyendo más del $70 \%$ total de los componentes marinos.

\section{Formación Puerto Madryn en la sección Puerto Pirámides}

En las muestras de la Formación Puerto Madryn se reconocieron 25 especies de quistes de dinoflagelados, acritarcos y algas prasinofíceas (Tasmanites sp.) con excelente grado de preservación (Figuras 4-6). Las frecuencias relativas de los palinomorfos continentales versus los palinomorfos marinos así como también las frecuencias relativas de los quistes de dinoflagelados, acritarcos y algas marinas (Figura 4) muestran variaciones a lo largo del perfil y marcan dos tramos bien diferenciados. El tramo inferior, de aproximadamente $m$ de espesor (S44 a S42), contiene las asociaciones con la mayor riqueza y abundancia de especies, con predominio de taxones neríticos y baja representación de taxones oceánicos, como Impagidinium y Nematosphaeropsis. Las asociaciones están compuestas mayoritariamente por el orden Gonyaulacales, principalmente por las especies Spiniferites/ Achomosphaera spp., Reticulatosphaera actinocoronata, Operculodinium centrocarpum y Batiacasphaera minuta. Las protoperidiniaceas están bien representadas, particularmente por el género Brigantedinium en la parte superior del tramo inferior y diversas especies de Selenopemphix, al igual que taxones indicadores de aguas frías, tales como Habibacysta tectata y Filisphaera filifera (Head, 1997). Estas asociaciones están en concordancia con un ambiente de depositación de plataforma interna, tal como fuera definido por del Río et al. (2001) y representarían los niveles de máxima inundación para toda la Formación Puerto Madryn. En el tramo superior (S46 a S19) se reduce notablemente el número de especies, quedando representadas mayoritariamente por Spiniferites y Operculodinium, con incremento en las proporciones de algas prasinofíceas y acritarcos. Estas asociaciones denotan condiciones de depositación marino marginal hacia la parte superior de la sección estudiada.

\section{EDAD BASADA EN LOS QUISTES DE DINOFLAGELADOS}

Las especies de los quistes de dinoflagelados halladas en las Formaciones Barranca Final y Puerto Madryn tienen, en general, rangos estratigráficos amplios. Por lo tanto, la edad relativa de estas dos unidades litoestratigráficas está basada en unas pocas especies, cuyos rangos estratigráficos son conocidos y están calibrados en el Hemisferio Norte (De Verteuil \& Norris, 1996; Williams et al., 2004, Louwye et al., 2007a,b). Los rangos de las tres especies diagnósticas, Labyrinthodinium truncatum, Habibacysta tectata y 

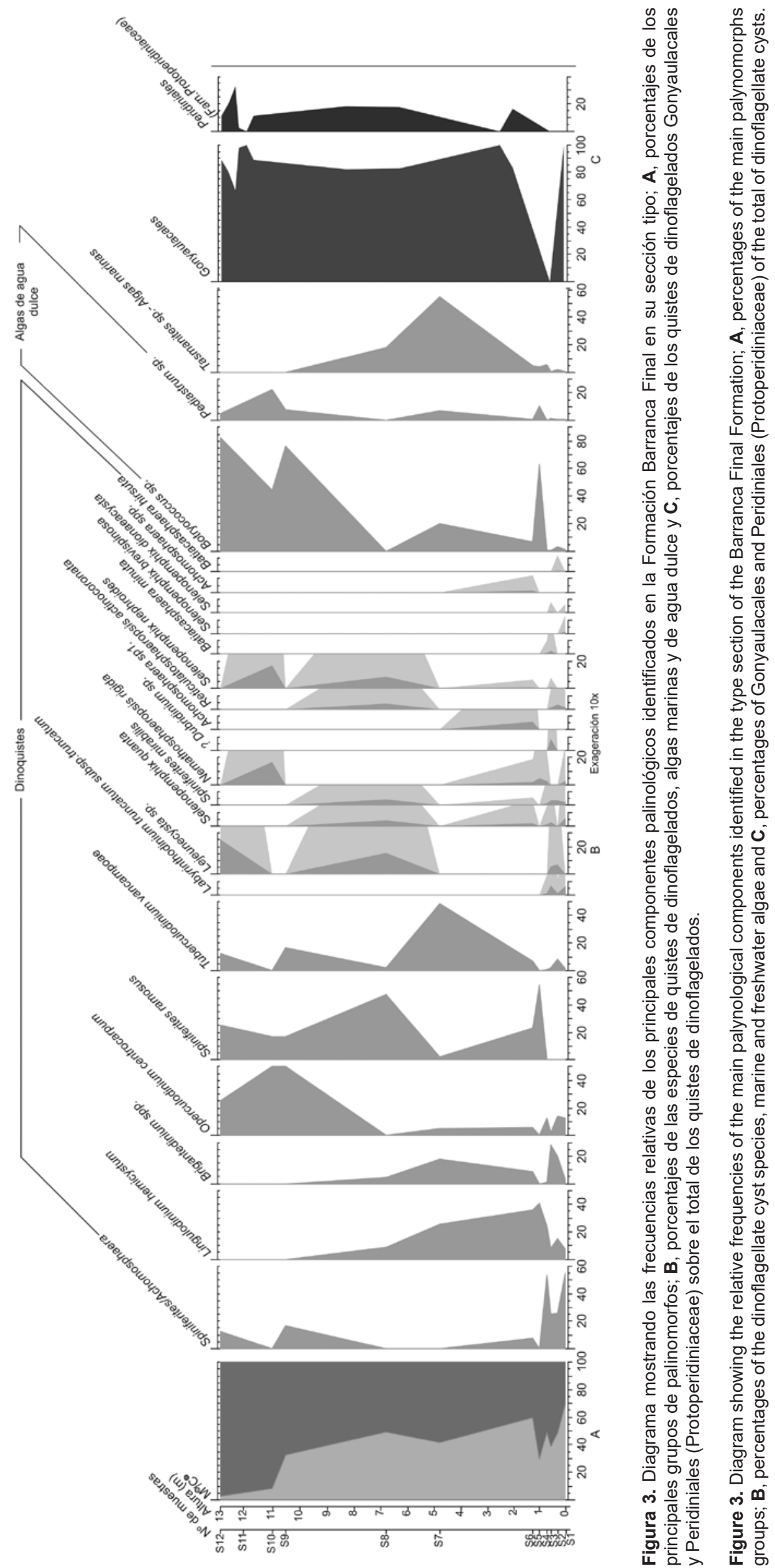


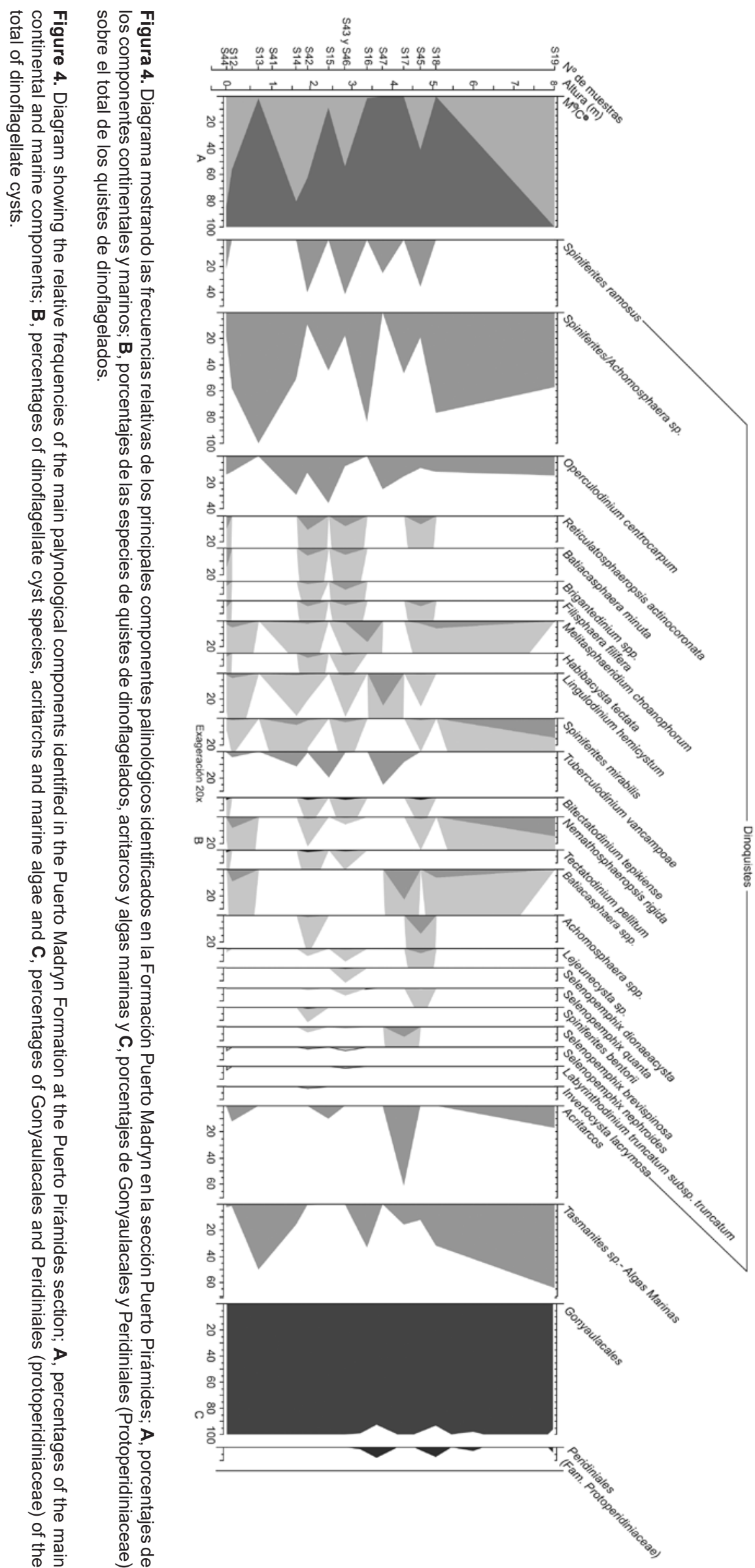



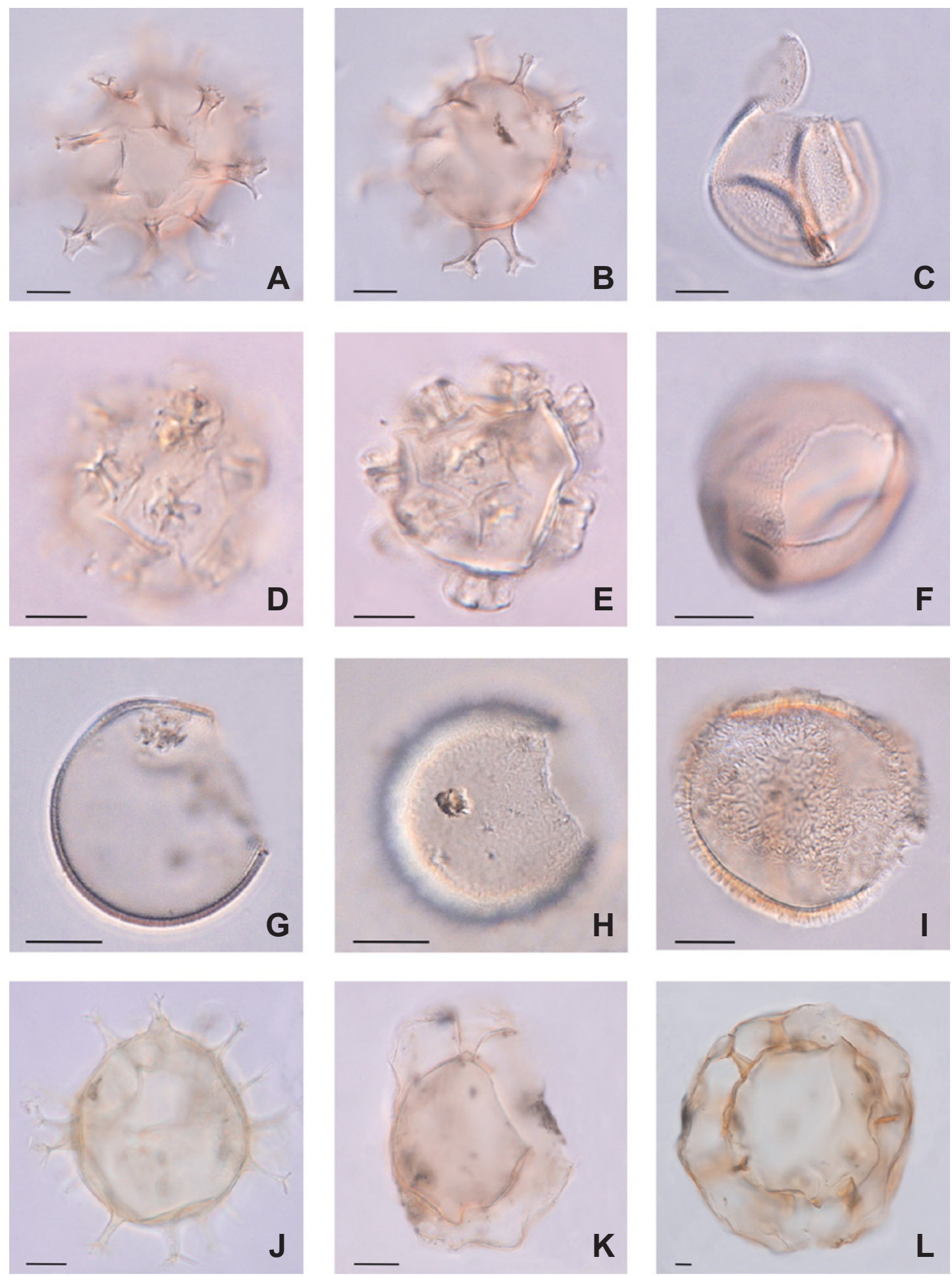

Figura 5. Quistes de dinoflagelados identificados en las formaciones Barranca Final y Puerto Madryn. A-B, Achomosphaera sp. (Head, 1994). A, vista ventral, foco alto; B, vista ventral, foco intermedio. C, F, Batiacasphaera minuta (Matsuoka, 1983); C, vista lateral, foco alto; F, vista apical, foco alto. D-E, Labyrinthodinium truncatum truncatum (Piasecki, 1980); D, vista apical, foco bajo; E, vista apical, foco alto. G-H, Habibacysta tectata (Head et al., 1989b); G, vista lateral, corte óptico; H, vista lateral, foco bajo. I, Filisphaera filifera (Bujak, 1984), vista ventral, foco alto intermedio. J, Spiniferites bentorii (Rossignol, 1964), vista dorsal, corte óptico. K, Invertocysta lacrimosa (Edwards, 1984), vista intermedia, foco bajo. L, Tuberculodinium vancampoae (Rossignol, 1962), vista antapical, foco alto. Escalas $=10 \mu \mathrm{m}$.

Figure 5. Dinoflagellate cysts identified in the Barranca Final and the Puerto Madryn formations. A-B, Achomosphaera sp. (Head, 1997). A, ventral view, high focus; B, ventral view, intermediate focus. C, F, Batiacasphaera minuta (Matsuoka, 1983); C, lateral view, high focus; F, apical view, high focus. D-E, Labyrinthodinium truncatum truncatum (Piasecki, 1980), D, apical view, low focus; E, apical view, high focus. G-H, Habibacysta tectata (Head et al., 1989b), G, lateral view, cross-section; H, lateral view, low focus. I, Filisphaera filifera (Bujak, 1984), ventral view, intermediate high focus. J, Spiniferites bentorii (Rossignol, 1964), dorsal view, cross-section. K, Invertocysta lacrimosa (Edwards, 1984), intermediate view, low focus. L, Tuberculodinium vancampoae (Rossignol, 1962), antapical view, high focus. Scale bars $=10 \mu \mathrm{m}$. 

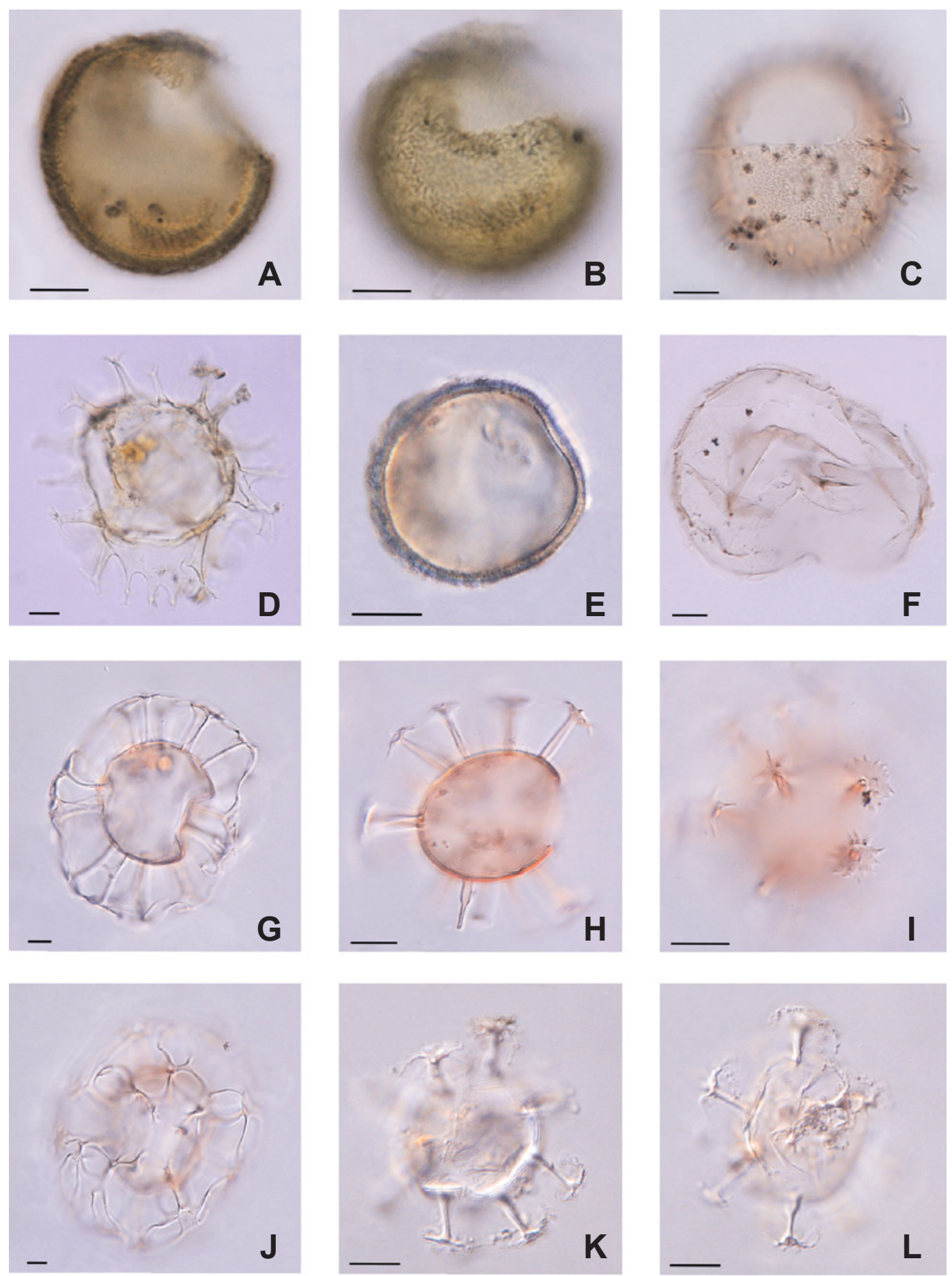

Figura 6. Quistes de dinoflagelados identificados en las formaciones Barranca Final y Puerto Madryn. A-B, Bitectatodinium tepikiense (Wilson, 1973); A, vista ventral oblicua, corte óptico; B, vista ventral oblicua, foco bajo. C, Operculodinium centrocarpum (Deflandre \& Cookson, 1955), vista dorsal, foco alto. D, Spiniferites mirabilis (Rossignol, 1964), vista ventral, foco bajo. E, Tectatodinium pellitum (Wall, 1967), vista ventral, corte óptico. F, Selenopemphix nephroides (Benedek, 1972), Vista apical, foco intermedio. G, J, Nematosphaeropsis rigida (Wrenn, 1988); G, vista ventral, corte óptico; J, vista ventral, foco intermedio alto. H-I, Melitasphaeridium choanophorum (Deflandre \& Cookson, 1955); H, vista lateral derecha, corte óptico; I, vista lateral derecha, foco alto. K-L, Reticulatosphaera actinocoronata (Benedek, 1972), K, vista general, foco bajo; $\mathbf{L}$, vista general, foco alto. Escalas $=10 \mu \mathrm{m}$.

Figure 6. Dinoflagellate cysts identified in the Barranca Final and the Puerto Madryn formations. A-B, Bitectatodinium tepikiense (Wilson, 1973); A, ventral view oblique, cross-section; B, ventral view oblique, low view. C, Operculodinium centrocarpum (Deflandre \& Cookson, 1955), dorsal view, high focus. D, Spiniferites mirabilis (Rossignol, 1964), ventral view, low focus. E, Tectatodinium pellitum (Wall, 1967), Ventral view, cross-section. F, Selenopemphix sp. (Benedek, 1972), apical view, intermediate focus. G, J, Nematosphaeropsis rigida (Wrenn, 1988); G, ventral view, cross section; J, ventral view, intermediate high focus. H-I, Melitasphaeridium choanophorum (Deflandre \& Cookson, 1955); H, right lateral view, cross-section; I, right lateral view, high focus. K-L, Reticulatosphaera actinocoronata (Benedek, 1972), K, general view, low focus; L, general view, high focus. Scale bars $=10 \mu \mathrm{m}$. 
Reticulatosphaera actinocoronata, están en concordancia con las edades radiométricas conocidas para estos estratos (Scasso et al., 2001; Palazessi et al., 2014). La presencia de L. truncatum truncatum sugiere una edad comprendida entre el Mioceno medio y Mioceno tardío (Langiano-Tortoniano) para los depósitos marinos someros inferiores de la Formación Barranca final en su sección tipo. En la Formación Puerto Madryn la presencia de Habibacysta tectata y de Labyrinthodinium truncatum truncatum, indican para el tramo inferior de la sección Puerto Pirámides (S44 a S42) una edad comprendida entre el Mioceno medio tardío y Mioceno tardío (Serravaliano-Tortoniano). Williams et al. (2004) ubicaron la primera ocurrencia de L. truncatum truncatum a los 16,5 Ma y Louwye et al. (2007b) en el Burdigaliano tardío de la Cuenca Porcupine, Bélgica (en la base del Subcron 5Cn.2r). Por otra parte, Williams et al. (2004) consideraron la última ocurrencia de L. truncatum truncatum a los 7,85 y 9 Ma en latitudes medias y bajas del Hemisferio Norte, de acuerdo a De Verteuil \& Norris (1996) and Zevenboom (1995), respectivamente. La primera ocurrencia de Habibacysta tectata fue registrada a los 13,6 Ma según Louwye et al. (2007a) y a los $14 \mathrm{Ma}$ (Langiano tardío) según Williams et al. (2004). En la parte superior de la sección muestreada de la Formación Puerto Madryn, se observa la continentalización de la secuencia, con la consecuente disminución de la riqueza y abundancia de los quistes de dinoflagelados y la desaparición de las especies diagnósticas. No obstante, Reticulatosphaera actinocoronata marca una edad mínima zancleana para la parte superior, la última ocurrencia de Reticulatosphaera actinocoronata fue establecida a los 4,8 Ma, en la costa este de Canadá (Fensome et al., 2008).

\section{DISCUSIÓN}

La edad de la Formación Barranca Final ha sido controvertida desde la descripción original de la unidad por Kaasschieter $(1963,1965)$, con propuestas que sugirieron edades desde el Eoceno hasta el Plioceno, basadas en foraminíferos bentónicos y planctónicos, nanofósiles calcáreos, ostrácodos y palinomorfos principalmente en materiales provenientes del subsuelo de la cuenca del Colorado (cf. Malumián et al., 1998 y la discusión allí planteada). De acuerdo a Malumian et al. (1998), el rango amplio de edad relacionado a la Formación Barranca Final probablemente se deba a la indefinición de los limites estratigráficos entre unidades, principalmente el límites entre la Formación Barranca Final y la infrayacente Formación Elvira. Esta última unidad estratigráfica ha sido identificada como parte de la transgresión "Patagoniense" del Mioceno temprano, caracterizada por la zona de foraminíferos bentónicos de Cribrorotalia hornibrooki (Malumián et al., 1998) y portadora de una microfauna reconocida en la Formación Monte León (cuenca Austral) con edades de 22-18Ma (Parras et al., 2012). Ambas formaciones tienen composiciones microfaunísticas, florísticas y litológicas claramente distinguibles en el subsuelo de la Cuenca del Colorado. Sin embargo, rocas de la ingresión "Patagoniense" (Formación Elvira) fueron incluidas en la Formación Barranca Final (Malumián et al., 1998). Estos autores, reconocen a la Formación Barranca Final, en su localidad tipo, como portadora de microfósiles típicos de la transgresión "Entrerriense", y de una asociación de foraminíferos bénticos de la Zona de Protelphidium tuberculatum Biozone D'Orbigny, cuya edad está comprendida entre el Mioceno medio y tardío. Las asociaciones de quistes de dinoflagelados provenientes de los depósitos marinos someros de la parte inferior de la Formación Barranca final indican, en concordancia con los foraminíferos asociados, una edad comprendida entre el Langiano y Tortoniano (Guler et al., 2002), basada principalmente en el rango de la especie Labyrinthodinium truncatum truncatun. Recientemente, las tres edades isotópicas obtenidas a partir de valvas de ostras bien preservadas corresponden al Mioceno tardío $(9,61 ; 8,3$, y 6,48 Ma; Palazzesi et al., 2014). Las dos edades ${ }^{87} \mathrm{Sr} /{ }^{86} \mathrm{Sr}$ de 9,61 y 8,3 Ma permiten ajustar la edad de las asociaciones de quistes de dinoflagelados al Tortoniano, sugiriendo además, que sólo la parte superior del rango estratigráfico de L. truncatum truncatum estaría representado en la base de la Formación Barranca Final.

La edad ${ }^{87} \mathrm{Sr} /{ }^{86} \mathrm{Sr}$ de $6,48 \mathrm{Ma}$ (Palazzesi et al., 2014) está vinculada a niveles de la parte media baja de la sección, portadores de asociaciones caracterizadas por un incremento en las proporciones de palinomorfos continentales, incremento en las proporciones de algas clorifíceas y la disminución en la riqueza de especies de quistes de dinoflagelados, con la ausencia de indicadores biostratigráficos. La edad de la parte superior de la sección está acotada por la formación Río Negro que yace concordantemente a la Formación Barranca Final y fue datada en 4,41 Ma según el método de trazas de fisión (Alberdi et al., 1997).

La edad de la Formación Puerto Madryn ha sido discutida principalmente sobre la base de ostrácodos, foraminíferos y contenido de moluscos, y comprende el intervalo Mioceno medio a Mioceno tardío (ver discusión en del Río, 1988; del Rio et al., 2001; Scasso et al., 1999, 2001; Marengo, 2015 y referencias allí citadas). Análisis de ${ }^{87} \mathrm{Sr} /{ }^{86} \mathrm{Sr}$ en conchillas de pectínidos indicaron una edad Tortoniano medio (alrededor de $10 \mathrm{Ma}$ ) para los depósitos "Entrerrienses" correspondientes a la Formación Puerto Madryn (Scasso et al., 2001). La edad sugerida a partir de los quistes de dinoflagelados en la sección Puerto Pirámides, comprendida en el intervalo Serravaliano a Tortoniano (Mioceno medio tardío a Mioceno tardío), concuerda con las edades propuestas por la micro y macrofauna y es consistente con la edad de Mioceno tardío indicada por los estudios de ${ }^{87} \mathrm{Sr} /{ }^{86} \mathrm{Sr}$ de $10 \mathrm{Ma}$. No obstante, el intervalo analizado en este estudio abarca aproximadamente solo $8 \mathrm{~m}$ del total de la sección, que corresponden a los niveles máximos de inundación registrados para la Formación Puerto Madryn (e.g. del Río et al., 2001). En ambas unidades estaría representada la parte superior del rango de Labyrinthodinium truncatum truncatum, así como también existe una marcada afinidad en las asociaciones esporopolínicas recuperadas (Palazzesi \& Barreda, 2004).Las dataciones radiométricas (Palazzesi et al., 2014; Scasso et al., 2001) indican que estas unidades se depositaron en el Mioceno tardío, después de $10 \mathrm{Ma}$. No obstante, no es posible aun establecer a partir de 
los quistes de dinoflagelados una correlación precisa entre las Formaciones Puerto Madryn y Barranca Final. La escasa afinidad entre las asociaciones de quistes de dinoflagelados entre ambas formaciones se debe presumiblemente a la baja riqueza y el fuerte carácter marginal que exhibe la Formación Barranca Final.

\section{AGRADECIMIENTOS}

El presente trabajo es una contribución al proyecto PICT 2012-0911 "Diversidad de las floras de Patagonia durante el Eoceno-Mioceno y su relación con la inferida para otras paleolatitudes".

\section{REFERENCIAS}

Alberdi, M.T.; Bonadonna, F.P. \& Ortiz, E. 1997. Chronological correlation, paleoecology and paleogeography of the Late Cenozoic South American Rionegran Land-mammal fauna: a review. Revista Española de Paleontología, 12:249-255.

Caramés, A.; Malumián, N. \& Náñez, C. 2004. Foraminíferos del Paleogeno del Pozo Península Valdés (PV. es-1), Patagonia septentrional, Argentina. Ameghiniana, 4:461-474.

Cione, A.L.; Azpelicueta, M.M.; Casciotta, J.R. \& Dozo, M.T. 2005. Tropical freshwater teleosts from Miocene beds of eastern Patagonia, southern Argentina. Geobios, 38:29-42. doi:10.1016/j.geobios.2003.08.005

De Verteuil, L. \& Norris, G. 1996. Miocene dinoflagellate stratigraphy and systematics of Maryland and Virginia. Micropaleontology supplement, 42:1-172.

del Río, C.J. 1988. Bioestratigrafía y cronoestratigrafía de la Formación Puerto Madryn (Mioceno medio). Provincia del Chubut - Argentina. Anales de la Academia Nacional de Ciencias Exactas, Físicas y Naturales, 40:231-254.

del Río, C.J. 2000. Malacofaunas de las formaciones Paraná y Puerto Madryn (Mioceno marino, Argentina): su origen, composición y significado bioestratigráfico. In: F.G. Aceflolaza \& R. Herbst (eds.) El Neógeno de Argentina, Tucumán, INSUGEO, p. 77-101 (Serie Correlación Geológica 14).

del Río, C.J. 2004. Tertiary marine Molluscan Assemblages of Eastern Patagonia (Argentina): a biostratigraphic analysis. Journal of Paleontology, 78:1097-1122. doi:10.1666/00223360(2004)078<1097:TMMAOE >2.0.CO;2

del Río, C.J. \& Martínez, S.A. 1998. El Mioceno marino en la Argentina y en el Uruguay. In: C. J. Del Río (ed.). Moluscos marinos Miocenos de la Argentina y del Uruguay, Academia Nacional de Ciencias Exáctas Físicas y Naturales, p. 6-25.

del Río, C.J.; Martinez, S.A. \& Scasso, R.A. 2001. Nature an origin of spectacular marine Miocene Shell beds of northeastern Patagonia (Argentina): paleoecological and bathymetric significance. Palaios, 16:3-25. doi:10.1669/0883-1351(2001)016<0003:NA OOSM>2.0.CO;2

Dozo, M.T.; Bouza, P.; Monti, A.; Palazzesi, L.; Barreda, V.; Massaferro, G.; Scasso, R.A. \& Tambussi, C. 2010. Late Miocene continental biota in Northeastern Patagonia (Península Valdés, Chubut, Argentina). Palaeogeography, Palaeoclimatology, Palaeoecology, 297:100-109. doi:10.1016/j.palaeo.2010.07.018

Fensome, R.A.; MacRae, R.A. \& Williams, G.L. 2008. The Lentin and Williams Index of fossil dinoflagellates. Pittsburgh, American Association of Stratigraphic Palynologists. (Contributions Series 1).
Fensome, R.A. \& Williams, G.L. 2004. The Lentin and Williams Index of fossil dinoflagellates. Pittsburgh, American Association of Stratigraphic Palynologists, 909 p. (Contributions Series 42).

Feruglio, E. 1949. Descripción Geológica de la Patagonia. Buenos Aires, Dirección General de Yacimientos Petrolíferos Fiscales, $349 \mathrm{p}$.

Frenguelli, J. 1921. El Entrerriense del Golfo Nuevo en el Chubut: Córdoba. Boletín de la Academia Nacional de Ciencias de Córdoba, 29:191-270.

Gélos, E.M.; Spagnuolo, J.O. \& Schillizzi, R.A.1993. Las unidades morfológicas de la costa oeste del Golfo de San Matías y su evolución. Revista de la Asociación Geológica Argentina, 47:365-371.

Gradstein, F.M.; Ogg, J.G.; Schmitz, M.D. \& Ogg, G.M. 2012. The Geologic Time Scale 2012. Boston, Elsevier, 1144 p. doi:10.1016/ B978-0-444-59425-9.11001-5.

Grimm, E. 1991-2014. Tilia software. Springfield, Illinois State Museum. Resercha and collection Center.

Guler, M.V.; Guerstein, G.R. \& Archangelsky, S. 2002. Quistes de dinoflagelados del Cretácico inferior de la plataforma continental argentina: resultados bioestratigráficos. Revista del Museo Argentino de Ciencias Naturales, 5:225-233.

Haller, M. 1979. Estratigrafía de la región al poniente de Puerto Madryn. In: CONGRESO GEOLÓGICO ARGENTINO, 7, 1978. Actas, Neuquén, p. 285-297.

Head, M.J. 1997. Thermophilic dinoflagellate assemblages from the mid Pliocene of eastern England. Journal of Paleontology, 71:165-193. doi:10.1017/S0022336000039123

Kaasschieter, J.P.H. 1963. Geology of the Colorado Basin. Tulsa Geological Society Digest, 31: 177-187.

Kaasschieter, J.P.H. 1965. Geología de la Cuenca del Colorado. In: JORNADAS GEOLÓGICAS ARGENTINAS, 3, 1965. Actas, Tucumán, p. 251-271.

Louwye, S.; De Schepper, S.; Laga, P. \& Vandenberghe, N. 2007a. The Upper Miocene at the southern North Sea Basin: a palaeoenvironmental and stratigraphic reconstruction with dinoflagellate cysts. Geological Magazine, 144:33-52. doi:10.1017/S0016756806002627

Louwye, S.; Foubert, A.; Mertens, K. \& Van Rooij, D. 2007 b. Integrated stratigraphy and palaeoecology of the Lower and Middle Miocene of the Porcupine Basin. Geological magazine, 145:321-344. doi:10.1017/S0016756807004244

Malumián, N.; Suriano, J. M. \& Cobos, J. C. 1998. La Formación Barranca Final en su localidad tipo. Mioceno, Cuenca del Colorado. In: CONGRESO LATINOAMERICANO DE GEOLOGÍA, CONGRESO NACIONAL DE GEOLOGÍA ECONÓMICA, 10,6, 1998, Actas, Buenos Aires, p. 125-130.

Marengo, H. 2015. Neogene micropaleontology and stratigraphy of Argentina. The Chaco-Paranense Basin and the Peninsula de Valdés. New York, Springer, 218 p. doi:10.1007/978-3-319-12814-6

Masiuk, V.; Becker, D. \& García, E.N. 1976. Micropaleontología y sedimentología del Pozo YPF Ch.PV es-1 (Península de Valdez) Provincia del Chubut, República Argentina. Importancia y correlaciones. Buenos Aires, Yacimientos Petrolíferos Fiscales, 22 p. (ARPEL 24).

Palazzesi, L. \& Barreda, V. 2004. Primer registro palinológico de la Formación Puerto Madryn, Mioceno de la provincia del Chubut, Argentina. Ameghiniana, 41:355-362.

Palazzesi, L.; Barreda,V.D.; Cuitiño, J.I.; Guler, M.V.; Tellería, M.C. \& Ventura Santos, R. 2014. Fossil pollen records indicate that Patagonian desertification was not solely a consequence of Andean uplift. Nature Communications, 5:1-8. doi:10.1038/ncomms4558. 
Parras, A.; Dix, G.R. \& Griffin, M. 2012. Sr-isotope chronostratigraphy of Paleogene-Neogene marine deposits: Austral Basin, southern Patagonia, Argentina. Journal of South American Earth Sciences, 37:122-135. doi:10.1016/j.jsames.2012.02.007.

Pross, J. \& Brinkhuis, H. 2005. Organic-walled dinoflagellate cysts as paleoenvironmental indicators in the Paleogene; a synopsis of concepts. Paläontologische Zeitschrift, 79:53-59. doi:10.1007/ BF03021753

Scasso, R. \& del Río, C. J. 1987. Ambientes de sedimentación y proveniencia de la secuencia marina del Terciario Superior de la región de Península Valdés. Revista de la Asociación Geológica Argentina, 42:291-321.

Scasso, R.A.; del Río, C.J. \& Martínez, S.A. 1999a. El contacto "Entrerriense" "Patagoniense" en Península Valdés, examen de una discontinuidad. In: CONGRESO GEOLÓGICO ARGENTINO, 14, 1999. Resúmenes en Acta, Salta, p. 73.

Scasso, R.A.; Dozo M.T.; Cuitiño J.I. \& Bouza, P. 2012. Meandering tidal-fluvial channels and lag concentration of terrestrial vertebrates in the fluvialtidal transition of an ancient estuary in Patagonia. Latin American journal of sedimentology and basin analysis, 19:27-45.

Scasso, R.A.; McArthur, J.M.; del Río, C.J.; Martínez, S. \& Thirlwall, M.F. $2001 .{ }^{87} \mathrm{Sr} /{ }^{86} \mathrm{Sr}$ Late Miocene age of fossil molluscs in the "Entrerriense" of the Valdés Peninsula (Chubut, Argentina). Journal of South American Earth Sciences, 14:319-329. doi:10.1016/S0895-9811(01)00032-3

Sluijs, A.; Pross, J. \& Brinkhuis, H. 2005. From greenhouse to icehouse; organic walled dinoflagellate cysts as paleoenvironmental indicators in the Paleogene. Earth Science Reviews, 68:281-315. doi:10.1016/j.earscirev.2004.06.001

Stover, L.; Gorga, M.P.; Neely, S.T. \& Montoya, D. 1996. Toward optimizing the clinical utility of distortion product otoacoustic emission measurements. Journal of the Acoustical Society of America, 100:956-967.

Wall, D.; Dale, B.; Lohmann, G.P. \& Smith, W.K. 1977. The environmental and climatic distribution of dinoflagellate cysts in modern marine sediments from regions in the North and South Atlantic Oceans and adjacent areas. Marine Micropaleontology, 2:121-200.

Williams, G.L.; Brinkhuis, H.; Pearce, M.A.; Fensome, R.A. \& Weegink, J.W. 2004. Southern Ocean and global dinoflagellate cyst events compared: index events for the Late CretaceousNeogene. In: N.F. Exon; J.P. Kennett \& M.J. Malone (eds.) Proceedings of the Ocean Drilling Program, College Station, Ocean Drilling Program, p. 1-98 (Scientific Results 189). doi:10.2973/odp.proc.sr.189.107.2004

Zambrano, J.J. 1980. Comarca de la cuenca Cretácica del Colorado. In: SIMPOSIO DE GEOLOGÍA REGIONAL ARGENTINA, 2, 1980. Actas, Córdoba, p. 1033-1070.

Zevenboom, D., 1995. Dinoflagellate cysts from the Mediterranean Late Oligocene and Miocene. University of Utrecht, Tesis doctoral, $221 \mathrm{p}$.

Received in November, 2015; accepted in June, 2016. 
Apéndice 1. Lista taxonómica de las especies de dinoflagelados, acritarcos algas de agua dulce y algas marinas identificados en la sección Puerto Pirámides de la Formación Puerto Madryn (1) y en la sección tipo de la Formación Barranca Final (2).

Appendix 1. Taxonomic list of the dinoflagellate cyst species, acritarchs, freshwater and marine algae at Puerto Pirámides section from the Puerto Madryn Formation (1) and the type section of Barranca Final Formation (2).

Achomosphaera sp. (Head, 1997) (2) (Figuras 5A-B)

Achomosphaera spp. (1,2)

Batiacasphaera spp. (1)

Batiacasphaera hirsuta (Stover, 1977) (2)

Batiacasphaera minuta (Matsuoka, 1983b) Matsuoka \& Head, 1992 (1, 2) (Figuras 5C-F)

Bitectatodinium tepikiense (Wilson, 1973) (1, 2) (Figuras 6A-B)

Brigantedinium spp. (Reid, 1977) Lentin \&Williams, 1993 (1, 2)

? Dubridinium sp. (Reid, 1977) (2)

Filisphaera filifera (Bujak, 1984) (1) (Figura 5I)

Habibacysta tectata (Head et al., 1989b) (1) (Figuras 5G-H)

Impagidinium sp. (Stover \& Evitt, 1978)

Invertocysta lacrymosa (Edwards, 1984) (1) (Figura 5K)

Labyrinthodinium truncatum truncatum (Piasecki, 1980) (1, 2) (Figuras 5D-E)

Lejeunecysta spp. (Artzner \& Dörhöfer, 1978) $(1,2)$

Lingulodinium hemicystum (McMinn, 1991) (1,2)

Melitasphaeridium choanophorum (Deflandre \& Cookson, 1955) Harland \& Hill, 1979 (1) (Figuras 6H-I)

Nematosphaeropsis rigida (Wrenn, 1988) (1,2) (Figuras 6G-J)

Operculodinium centrocarpum (Deflandre \& Cookson, 1955) Wall, 1967 (1, 2) (Figura 6C)

Reticulatosphaera actinocoronata (Benedek, 1972) Bujak \& Matsuoka, $1986(1,2)$ (Figuras 6K-L)

Selenopemphix brevispinosa (Head et al., 1989c) $(1,2)$

Selenopemphix dionaeacysta (Head et al., 1989b) $(1,2)$

Selenopemphix quanta (Bradford, 1975) Matsuoka, 1985a (1, 2)

Selenopemphix nephroides (Benedek, 1972) (1, 2) (Figura 6, F)

Spiniferites mirabilis (Rossignol, 1964) Sarjeant, 1970 (1, 2) (Figura 6D)

Spiniferites ramosus (Ehrenberg, 1838) Mantell, $1854(1,2)$

Spiniferites bentorii (Rossignol, 1964) Wall \& Dale, 1970 (1) (Figura 5J)

Spiniferites/Achomosphaera spp. (1,2)

Tectatodinium pellitum (Wall, 1967) (1) (Figura 6E)

Tuberculodinium vancampoae (Rossignol, 1962) Wall, 1967 (1,2) (Figura 5L)

Algas marinas

Tasmanites sp. (1,2)

Algas de agua dulce

Botryococcus sp. (2)

Pediastrum sp. (2)

Acritarcos sp. (1) 